\title{
Promelanogenic Effects by an Annurca Apple-Based Natural Formulation in Human Primary Melanocytes
}

This article was published in the following Dove Press journal: Clinical, Cosmetic and Investigational Dermatology

\author{
Maria Grazia Ferraro' \\ Marialuisa Piccolo' \\ Alessandro Pezzella $\left(\mathbb{D}^{2}\right.$ \\ Fabrizia Guerra' \\ Francesco Maione' \\ Gian Carlo Tenore' \\ Rita Santamaria' \\ Carlo Irace $\mathbb{D I}^{\prime}$ \\ Ettore Novellino'
}

'Department of Pharmacy, School of Medicine and Surgery, University of Naples "Federico II", Naples, 80I3I, Italy;

${ }^{2}$ Department of Chemical Sciences, University of Naples "Federico II", Naples, 80126 , Italy
Correspondence: Carlo Irace;

\section{Rita Santamaria}

Department of Pharmacy, School of Medicine and Surgery, University of Naples "Federico II", Via D. Montesano

49, Naples, 80 I3I, Italy

$\mathrm{Tel}+39081678416 ;+3908167842$ I

Fax +39 08। 678403

Email carlo.irace@unina.it; rita.

santamaria@unina.it
Introduction: Melanocytes are engaged in synthesis, transport, and release of pigments at the epidermal-melanin units in response to the finely regulated melanogenic pathway. A multifaceted combination of both intrinsic and extrinsic factors - from endocrine and paracrine dynamics to exogenous stimuli such as sunlight and xenobiotics - modulates expression and activity of proteins involved in pigmentation, including the rate-limiting enzyme tyrosinase. As well as playing critical physiological functions comprising skin photoprotection, melanins define hair and skin pigmentation which in turn have impacted considerably to human social communication since time immemorial. Additionally, numerous skin diseases based on pigmentation alterations can have serious public influence. While several melanogenesis inhibitors are already available, the number of melanin activators and tyrosinase stimulators as drug-like agents is still limited.

Methods: To explore the biological effects of an Annurca Apple-based nutraceutical preparation (AMS) on melanin production, experiments in cellular models of human skin were performed. Both primary cultures and co-cultures of epidermal melanocytes (HEMa) and follicular keratinocytes (HHFK) were used.

Results: We show that AMS, by now branded for its cutaneous beneficial effects, induces in total biocompatibility a significant promelanogenic effect in human primary melanocytes. In line, we found melanin cytosolic accumulation consistent with tyrosinase up-regulation.

Conclusion: Disposal of skin pigmenting agents would be attractive for the treatment of hypopigmentation disorders, to postpone skin photoaging or simply for fashion, so that discovery and development of melanogenesis stimulators, especially from natural sources, is nowadays a dynamic area of research.

Keywords: melanins, skin pigmentation, tyrosinase, skin pigmenting agents, natural bioactive molecules

\section{Introduction}

According to the latest evidence, melanin consists of an insoluble high molecularweight polymer involving 5,6-dihydroxyindole (DHI), produced through a complex pathway starting from tyrosine. In turn, based on several factors both endogenous and exogenous, hydroxyindole monomer units can occur in diverse redox status as well as hydrated molecular forms. ${ }^{1-4}$ The result is a rather intricate biopolymer, exhibiting a variety of biological properties. ${ }^{2,5,6}$ Indeed, melanin is currently a standard name used to indicate a family of dark pigments endowed with several biological functions, comprising mainly skin and hair pigmentation. 
Their molecular structure is well matched to block biocidal ultraviolet (UV) radiation and to adsorb visible light. ${ }^{7}$ Colour variation and distribution in human skin and hair reflect the type and amount of produced melanins. ${ }^{8}$ Moreover, by their selective binding, cutaneous pigment can provide for detoxification of chemicals, toxins, and heavy metals from the body. ${ }^{2}$ The functional meaning of melanins is intimately connected with the metabolic activity of neurocrest-derived dendritic cells, ie, melanocytes, endowed with definite melanogenetic compartments identified in turn as melanosomes. ${ }^{9,10}$ In the basal layers of epidermis, melanic pigments are produced in melanin-epidermal functional units, where melanocytes are associated with undifferentiated keratinocytes, which can acquire granules of mature melanin by cytocrine secretion in a dendritic-based process. ${ }^{11}$ Besides in skin and hair follicles, in human melanocytes are found in eyes, inner ear, bones, nervous and cardiovascular systems, and even in adipose tissues. ${ }^{2}$ There are two types of melanic pigmentation in humans: the constitutional one, genetically determined in the absence of sunlight exposure or other influences, and the optional one, induced by sunlight exposure (tan). Concerning the constitutional pigmentation, as well as being regulated by hormones and nervous system, other intrinsic factors may have a significant impact on melanin production, such as ethnicity and age, but also inflammation and a variety of pathophysiological conditions. ${ }^{1,12-14}$ Intrinsic factors include several molecules and cytokines resulting from surrounding keratinocytes and dermal fibroblasts, but also from endocrine, neural and inflammatory cells which are in turn responsive to hormones. ${ }^{15}$ In this context, based on recent evidence, skin response to such an intricate combination of stimulatory factors is believed to be mediated by a cutaneous neuroendocrine system ${ }^{16,17}$ In addition, exogenous stimuli other than UV radiation can influence melanogenesis, including xenobiotics which can impact either directly or indirectly on melanin synthesis and accumulation, largely by controlling expression and activity of proteins engaged in pigmentation. ${ }^{15,18}$ Just in recent years, melanin stimulators from natural and synthetic sources - to be hypothetically developed as skin pigmentation agents - have been the subject of intense investigations. ${ }^{19-21}$ Cosmetic tan stimulators with different mechanisms of action are already available to the market and widely used, but they give rise to subjective darkening complexion effects and not always they are fully examined products. Various side-effects have been in fact ascribed to these active cosmetic ingredients. ${ }^{22}$ Since ever skin and hair pigmentation have impacted considerably to human overall physical appearance and to social/sexual communication, so that melanin-browning reactions are today highlights for both cosmetic and pharmaceutical industries. Several bioactive molecules of natural or synthetic origin, as well as mixtures such as plant extracts, are exploited therapeutically for numerous skin disorders involving depigmentation, from the most trivial cases to the most devastating ones such as vitiligo. ${ }^{20,23}$ Furthermore, studies are also ongoing to develop plant-derived materials to stimulate promelanogenic effects for UV-induced skin damage protection. ${ }^{24}$ Indeed, due to their plenty of sources and safety, traditional herbs, plants, and fruits are broadly exploited for the treatment of skin hypopigmentation. In this frame, we recently proved by preclinical investigations that an Annurca Apple-based nutraceutical preparation (AMS) - rich in natural polyphenols relating to the wider class of bioflavonoids - acts on definite skin cells by considerably stimulating the production of cytokeratins. ${ }^{25,26}$ In line, clinical studies demonstrated that oral consumption of AMS as dietary supplement is endowed with a considerable hairinductive activity (trichogenicity), promptly improving hair and skin quality. ${ }^{26}$ Interestingly, throughout trials, many patients have experienced surprising hair repigmentation effects. Related biological effects had already been observed and reported in former clinical trials aimed at the evaluation of the beneficial effects of AMS on lipid metabolism. ${ }^{27,28}$ Not by chance, it has been described that some polyphenols are able to stimulate melanogenesis in in vitro models by different molecular mechanisms. ${ }^{21,29,30}$ Therefore, based on this evidence and considering the propensity of the AMS polyphenolic nutraceutical to exert biological effects at the skin level, here we have undertaken a study to deepen its effects on the production and accumulation of melanic pigments, performed by using primary in vitro models of human skin. For advanced preclinical investigations, melanocytes were ad hoc selected as directly engaged in melanin biogenesis, alone or together with epidermal (follicular) keratinocytes to establish the so-called melaninepidermal functional units. For this reason, keratinocytes/melanocytes primary co-culture models, replicating cell-cell interaction and crosstalk in the human skin, were additionally set up to better uncover the effects of the nutraceutical formulation in skin pigmentation. 


\section{Materials and Methods}

\section{Apple Collection}

Annurca apple fruits (M. pumila Miller cv Annurca) come from Valle di Maddaloni (Caserta, Italy). Once collected in October (green peel), they were reddened for about a month by means of the typical treatment, and then used as described below after careful analysis. ${ }^{25}$

\section{Preparation of Annurca-Based} Nutraceutical Formulation (AnnurMetS, AMS)

Annurca apples were subjected to a water extraction procedure. After filtration and centrifugation, the total aqueous extract has been concentrated and finally spray-dried in mixture with maltodextrins, achieving a fine powder termed AnnurMetS (AMS) and branded as AnnurtriComplex ${ }^{\circledR} .{ }^{25}$ The supplement, formulated by the Department of Pharmacy, University of Naples "Federico II" (Naples, Italy), is produced on large-scale by MB-Med Company (Turin, Italy).

\section{Primary Human Epidermal Melanocytes (HEMa)}

To explore AMS effects in preclinic, targeted experiments in vitro were conceived in primary cellular models of human skin. These cellular models derive directly from human biopsies acquired from specific skin regions (typically occipital and temporal areas of the head). In particular, primary cultures of human epidermal melanocytes (normal human epidermal melanocytes HEMa) engaged in melanin production, purchased from ATCC $\left(\mathrm{ATCC}^{\circledR}\right.$, PCS-200-013 ${ }^{\mathrm{TM}}$, Manassas, VA, USA), were obtained by specific protocols performed on biological samples deriving from surgical procedures (human scalp biopsies, ATCC ${ }^{\circledR}$ PCS-200-013, Batch 63285932), carried out on a 40-year-old Caucasian white female donor. Once set up the culture, HEMa were grown in Dermal Cell Basal Medium (ATCC ${ }^{\circledR}$ PCS-200-030 ${ }^{\mathrm{TM}}$ ) complemented with Adult Melanocyte Growth Kit (ATCC PCS-200-042 ${ }^{\mathrm{TM}}$ ), according to ATCC recommendations, in a humidified $5 \%$ carbon dioxide atmosphere at $37^{\circ} \mathrm{C}$. For this research, primary melanocytes between passages 3 and 6 were employed.

\section{Primary Human Epidermal Follicular Keratinocytes (HHFK)}

Epidermal follicular keratinocytes (Human Hair Follicular Keratinocytes, HHFK), participating in hair biogenesis, have been acquired from ScienCell (ScienCell $^{\mathrm{TM}}$ Research Laboratories, Carlsbad, CA, USA). Cultures were obtained through definite dissection and dissociation approaches next to surgical procedures (human scalp biopsies, ScienCell ${ }^{\circledR}$ \# 2440, TAN Record \# 944) on appropriately donor selected based on precise phenotypic requirements (Caucasian race, male gender, age 55 years). HHFK cells were cultured in Keratinocyte Medium (KM, ScienCell ${ }^{\circledR}$ Cat. No. 2101) supplemented with $1 \%$ of keratinocyte growth supplement (KGS, ScienCell ${ }^{\circledR}$ Cat. No. 2152) and $1 \%$ of penicillin/streptomycin solution (P/S, ScienCell ${ }^{\mathbb{B}}$ Cat. No. 0503). According to ScienCell recommendations, cultures were maintained in a humidified $5 \%$ carbon dioxide atmosphere at $37^{\circ} \mathrm{C}$.

\section{Co-Cultures of Human Hair Follicular Keratinocytes and Epidermal Melanocytes} Co-cultures have been set up to study the interactions concerning different cell types. Two-dimensional (2D) direct coculture method in cell culture plates - helpful to investigate connections requiring direct contact (juxtacrine) and/or based on diffusion of soluble factors (paracrine) - has here been applied. ${ }^{25}$ In particular, for co-culture, HHFK cells were trypsinized and mixed with HEMa cells (also previously trypsinized) at $1: 1$ cell density ratio. The mixed cells $(1 \times$ $10^{6}$ cells) were immediately plated in $100 \times 20 \mathrm{~mm}$ Petri culture dish (Corning Incorporated, Falcon ${ }^{\circledR}$, NY, USA) and allowed to growth for $48 \mathrm{~h}$.

\section{Bioscreens in vitro}

Preclinic bioscreens were accomplished to study AMS bioactivity in human epidermal melanocytes (HEMa), in hair follicular epidermal keratinocytes (HHFK cells), and in cell co-culture system (HEMa and HHFK). Cell viability and proliferation were estimated after time- and concentrationdependent incubations with the nutraceutical formulation (up to $96 \mathrm{~h}$ with concentrations ranging from 0.5 to $3 \mathrm{mg} / \mathrm{mL}$ ). The experimental methodology was based on the evaluation of a "Cell survival index," resulting from the combination of cell viability with cell count, producing a more reliable benchmark of cellular responses in vitro. ${ }^{31}$ For this purpose, HEMa, HHFK and co-cultures were seeded in 96-microwell culture plates at a density of $10^{4}$ cells/well. After $24 \mathrm{~h}$ of growth, the culture medium was supplanted with new medium and cells were then incubated for 24, 48, 72 and 96 $\mathrm{h}$ with various concentrations of AMS. Cell viability was estimated using the MTT assay protocol, based on the redox ability of living mitochondria to convert the yellow 
3-(4,5-dimethyl-2-thiazolyl)-2,5-diphenyl-2H-tetrazolium bromide (MTT, Sigma-Aldrich, St. Louis, MO, USA) into insoluble purple formazan by mitochondrial dehydrogenase activity. Cell number was analysed by the TC20 automated cell counter (Bio-Rad, Hercules, CA, USA), providing an accurate and reproducible total count of cells and a live/dead ratio in one step by a specific dye (trypan blue, Bio-Rad, Hercules, CA, USA) exclusion assay, as described by us. ${ }^{31}$ Total counts and live/dead ratio from random cellular samples were subjected to comparisons with manual haemocytometers in control experiments.

\section{Cytomorphological Analysis}

HEMa, HHFK and co-culture systems (HEMa and HHFK) were cultured on $60 \mathrm{~mm}$ culture dishes by seeding $5 \times 10^{5}$ cells. At sub confluence, cells were treated with $3 \mathrm{mg} / \mathrm{mL}$ of AMS for $96 \mathrm{~h}$ (endpoint) in the same experimental conditions illustrated for bioscreens. After incubation, cultures were analyzed by a phase-contrast microscope to monitor any cytomorphological changes (Leizt Labovert inverted microscope, Leica Microsystems, Wetzlar, Germany). 200 $\times$ total magnification microphotographs $(20 \times$ objective and $10 \times$ eyepiece) were obtained by a standard VCR camera (Nikon Corporation, Tokyo, Japan). ${ }^{25}$

\section{UV Source and Irradiation}

Monolayers of HEMa or HEMa-HHFK co-cultures maintained as described above were irradiated with UV-B radiation using a Philips TL 20W/12 lamp $\left(5.4 \mathrm{~W} / \mathrm{m}^{2}\right)$, endowed with a wide emission spectrum ranging from 275 to $375 \mathrm{~nm}$ but emitting mainly between 290 and $320 \mathrm{~nm}$ with an optimum at $310 \mathrm{~nm}$. It emits $61 \%$ of its radiant energy in the UVB region of $290-320 \mathrm{~nm}, 6.1 \%$ in the low UVB range (280-290 nm), some (0.3-0.5\%) UVC below $280 \mathrm{~nm}$ (Philips, Eindhoven, The Netherlands). Before UV irradiation, cells were washed and covered with phosphate-buffered saline (PBS). The intensity of irradiation, measured with a UV meter (Spectrolyne mod., Spectronics Corp., Westbury, NY, USA) was $0.8 \mathrm{~mW} / \mathrm{cm}^{2}$ for UVB. ${ }^{32}$ For each irradiation session, cell monolayers received the non-cytotoxic $40 \mathrm{~mJ} / \mathrm{cm}^{2}$ dose of UVB. ${ }^{33}$

\section{DHI Autoxidation and Melanin Formation by Spectrophotometric Assay}

Time-dependent assays and progress curves were carried out using a $6 \mathrm{mg} / 100 \mathrm{~mL}$ DHI solution in $0.1 \mathrm{M}$ phosphate buffer at $\mathrm{pH}$ 7.0. When appropriate, $0.6 \mathrm{mg} / 100 \mathrm{~mL}$ of apple extract or quercetin were added to the mixture. Autoxidation of DHI was allowed to run at standard conditions and spectra were recorded at time interval of 60 minutes up to 24 hours and at 48 hours by using a JASCO V 730 diode array spectrophotometer (JASCO International Co. Ltd., Tokyo, Japan)

\section{Cellular Total Melanin Content Assay}

Total melanin levels were investigated according to the modified methods of Hosoi et al (1985). ${ }^{34}$ The quantitative determination of the intracellular content of melanins was performed via a spectrophotometric method after cell pellet lysis procedures and pigment isolation and purification. To generate calibration curves and to allow for an accurate quantitative analysis, a human synthetic melanin commercially available was used (Sigma-Aldrich, St. Louis, MO, USA). HEMa cells and co-culture with HHFK cells were cultured at $1 \times 10^{6}$ cells/dish. After $24 \mathrm{~h}$, cells were incubated with different concentrations of $\operatorname{AMS}(1,2$ and $3 \mathrm{mg} / \mathrm{mL}$ ) for $24 \mathrm{~h}$ or UV-B irradiated. After PBS washing, cells were harvested by trypsinization. The cell pellets were solubilized in $100 \mu \mathrm{L}$ of $1 \mathrm{~N} \mathrm{NaOH}$ at $37^{\circ} \mathrm{C}$ for 30 min. Standard solutions of melanin were incubated in parallel. Absorbance of samples was spectroscopically recorded at $450 \mathrm{~nm}$ and results were normalized based on the total cell number.

\section{Preparation of Cell Extracts and Tyrosinase Expression by}

\section{Immunodetection}

HEMa, HHFK and co-cultures were cultured in standard plastic $60 \mathrm{~mm}$ culture dishes by plating $5 \times 10^{5}$ cells. Behind reaching the subconfluence, cells were incubated with AMS as described above. After treatments, cells were washed and collected by scraping with PBS containing 1 mM EDTA and low-speed centrifugation. Cell pellets were then lysed at $4^{\circ} \mathrm{C}$ for $30 \mathrm{~min}$ in a buffer containing $20 \mathrm{mM}$ Tris- $\mathrm{HCl}, \mathrm{pH} 7.4,150 \mathrm{mM} \mathrm{NaCl}, 5 \mathrm{mM}$ EDTA, 5\% (v/v) glycerol, $10 \mathrm{mM} \mathrm{NP-40}$ and protease inhibitor tablets (Roche Molecular Systems, Risch, Switzerland). ${ }^{35}$ The supernatant fraction was obtained by centrifugation at $15,000 \times \mathrm{g}$ for $10 \mathrm{~min}$ at $4{ }^{\circ} \mathrm{C}$ and then stored at $-80^{\circ} \mathrm{C}$. Protein concentration was determined by the Bio-Rad protein assay (Bio-Rad, Hercules, CA, USA). To study protein expression by Western blot analysis, precise protein quantities $(50 \mu \mathrm{g})$ from cellular extracts were subjected to electrophoresis on 10\% SDS-PAGE and electrotransfer on $0.2 \mu \mathrm{m}$ nitrocellulose membranes (Trans-Blot ${ }^{\mathbb{B}}$ TurboTM, 
Transfer Pack, Bio-Rad, Hercules, CA, USA) by means of a Bio-Rad Transblot (Bio-Rad, Hercules, CA, USA). After reversible staining with Ponceau-S solution for protein visualization, membranes were blocked in milk buffer at room temperature $(1 \times \mathrm{PBS}, 5-10 \mathrm{~g} / 100 \mathrm{~mL}$ nonfat dry milk, $0.2 \% \mathrm{~g} / 100 \mathrm{~mL}$ Tween-20). For tyrosinase immunodetection, membranes were incubated at $4^{\circ} \mathrm{C}$ overnight with 1:250 monoclonal tyrosinase antibody (Invitrogen, Thermo Fisher Scientific, Waltham, MA, USA). A goat anti-mouse IgG+IgM (Jackson ImmunoResearch Laboratories, West Grove, PA, USA) 1:5000 was utilized as secondary antibody. The resulting immunocomplexes, highlighted by the ECL chemiluminescence procedure (ClarityTM Western ECL Substrate, Bio-Rad, Hercules, CA, USA), were examined by an imaging system (ChemiDoc Imaging System, Bio-Rad, Hercules, CA, USA) ${ }^{36}$ The GAPDH antibody (Sigma-Aldrich, St. Louis, MO, USA) was selected as housekeeping gene to control the results.

\section{Statistical Analysis}

The statistical analysis throughout experiments was accomplished by Graph-Pad Prism (Graph-Pad software Inc., San Diego, CA, USA). Experimental data were shown as mean values \pm SEM.

\section{Results}

\section{Bioscreen for AMS Biological Effects in Human Primary Melanocytes and Melanocytes/Keratinocytes Co-Cultures}

We first assessed the effects of AMS nutraceutical preparation on primary human melanocytes by setting up specific bioscreens at different concentrations $(0.5-3 \mathrm{mg} /$ $\mathrm{mL})$ and times $(24,48,72$, and $96 \mathrm{~h})$ to analyse cellular responses in terms of growth and vitality. The results reported as "Cell survival index" in Figure 1A-D by concentration-effect curves show no significant variation compared to untreated melanocytes cultured in the same experimental conditions. Therefore, the nutraceutical does not interfere with dynamic mechanisms underlying the regulation of cell proliferation. In addition, no decreasing trend in cell survival is noted at higher concentrations and for longer times (72 h), an indication of safety in use and biocompatibility. The screen was extended to both follicular keratinocytes and co-culture systems of melanocytes and keratinocytes. These data are in line with our previous results in human preclinical models of skin, suggesting the preparation would be safe and free of side effects. $^{25,26}$ A moderate increase in cell proliferation occurs just in melanocytes-keratinocytes co-cultures, here used to set up in vitro melanin-epidermal functional units. This effect is probably linked to a mutual stimulation of the two cell types during culture and treatments. In addition, we analysed cellular structure and morphology throughout bioscreens in vitro to monitor any significant changes. Following treatments, endpoints imaging by phase-contrast light microscopy were taken from single or mixed cell monolayers exposed for 48 and $96 \mathrm{~h}$ to 2 and $3 \mathrm{mg} / \mathrm{mL}$ of AMS. Microphotographs in Figure 1E well support the AMS biocompatibility in all the experimental conditions, by excluding interference with cell viability. Indeed, no cytomorphological alteration is detectable, nor hallmarks of suffering and/or activation of cell death pathways are visible after long incubation times and at the higher AMS concentrations.

\section{AMS Significantly Increases the Total Melanin Content in in vitro Models}

After having tested the biocompatibility of the nutraceutical supplement and its safety over a wide range of concentrations in preclinical models of human skin, we then investigated its biological effects on the biogenesis of melanic pigments in the same experimental conditions. We assessed the effects of AMS on the melanin production in primary cultures of human melanocytes (HEMa), and then in co-cultures with epidermal keratinocytes (HHFK) to reproduce an in vitro model reflecting interactions and crosstalk with the main skin cytotype. Next to in vitro treatments, the quantitative determination of the intracellular content of melanins was performed via a spectrophotometric method after cell lysis procedures, and pigment isolation and purification, as described in the experimental section. To generate calibration curves and to allow for an accurate quantitative analysis, a commercially available human synthetic melanin was used. As clearly shown in Figure 2A and B, melanocyte incubation with $0.5,1,2$, and $3 \mathrm{mg} / \mathrm{mL}$ of AMS for 48 (a) and $96 \mathrm{~h}$ (b) resulted in a remarkable increase in melanin content. This effect was concentration-dependent and, evidently, timedependent due to the accumulation of melanin granules in melanogenetic compartments. After $96 \mathrm{~h}$ of exposure to AMS at a concentration of $3 \mathrm{mg} / \mathrm{mL}$, the total quantity of melanins resulted over 1.5-fold higher compared to that of control melanocytes. Interestingly, the reported AMSdependent upsurge in melanin production and accumulation 
A

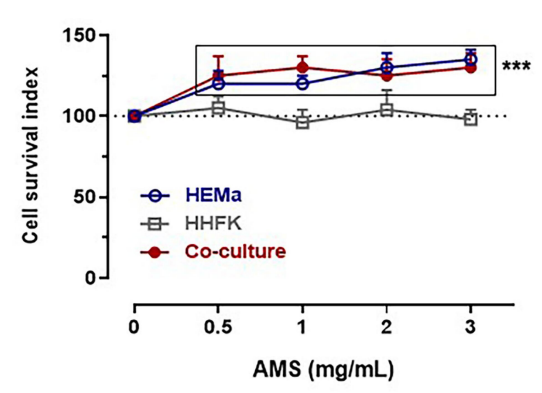

C

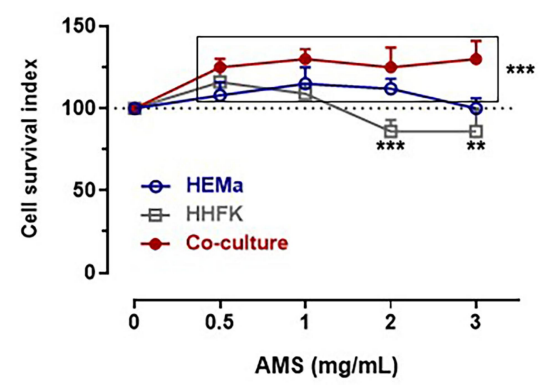

B

$48 \mathrm{~h}$

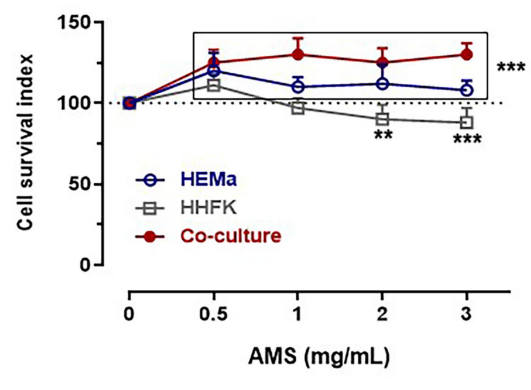

D

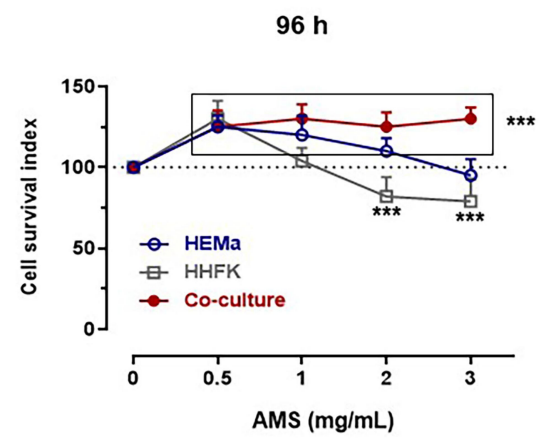

E

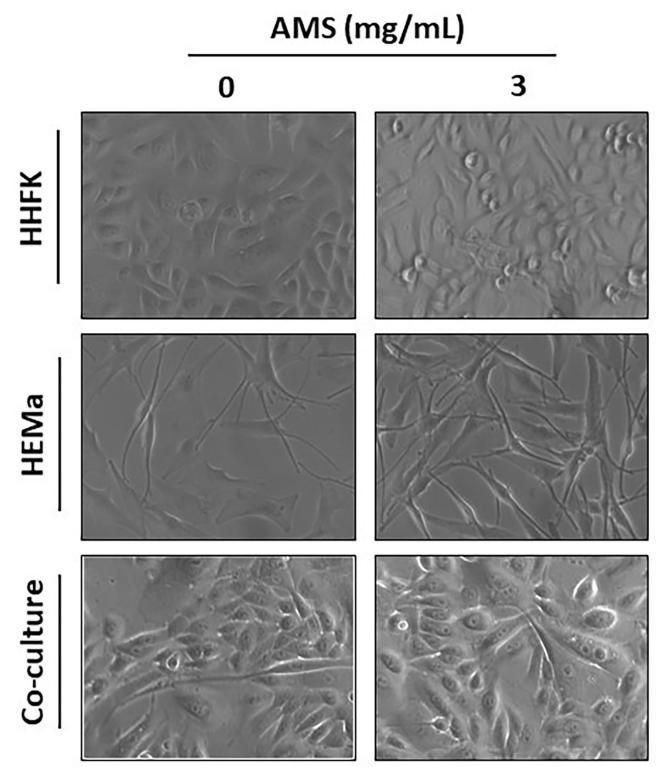

Figure I Preclinical bioscreen to estimate cellular response to AMS treatment. "Cell survival index" for HEMa, HHFK and HEMa-HHFK co-culture systems following 24 (A), 48 (B), 72 (C) and $96 \mathrm{~h}$ (D) of treatment with the indicated concentrations $(0.5 \div 3 \mathrm{mg} / \mathrm{mL})$ of AMS supplement. Results are expressed in line graphs as percentage of untreated control cells and are reported as mean of four independent experiments \pm SEM $(n=20)$. **** $<<0.00 \mathrm{I}$ vs control (untreated cells); ** $p<0.0 \mathrm{I}$ vs control (untreated cells). (E) Microphotographs by phase-contrast light microscopy at a $100 \times$ magnification ( $10 \times$ objective and a $10 \times$ eyepiece) of $\mathrm{HHFK}$ and $\mathrm{HEMa}$ cell monolayers, and HEMa-HHFK co-cultures for cytomorphological changes analysis at end-points $(96 \mathrm{~h}$ ) after incubation with $3 \mathrm{mg} / \mathrm{mL}$ of AMS nutraceutical, as indicated. The shown images are representative of four independent experiments.

was very similar to that observed after stimulation in vitro by melanogenetic UV-B radiation at the non-cytotoxic $40 \mathrm{~mJ} / \mathrm{cm}^{2}$ dose, as the main factor responsible for melanogenesis (approximately 2.5-fold increase at $96 \mathrm{~h}$ after stimulation compared to control melanocytes). In these experimental conditions, no UV-dependent effects were detected on melanocytes proliferation. In addition, the promelanogenic effect and the resulting pigment accumulation following AMS treatment 
A

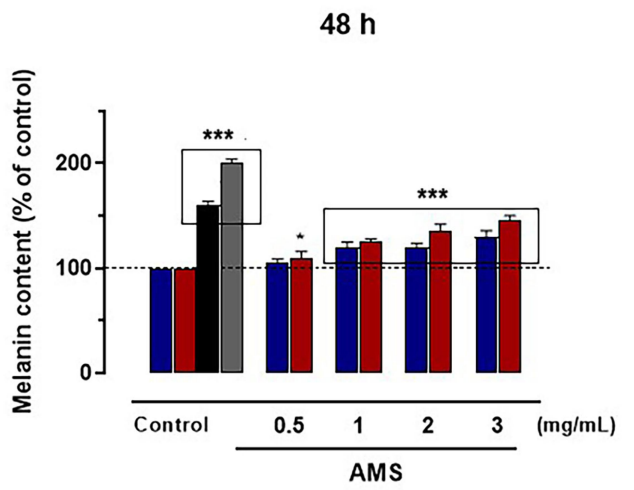

B

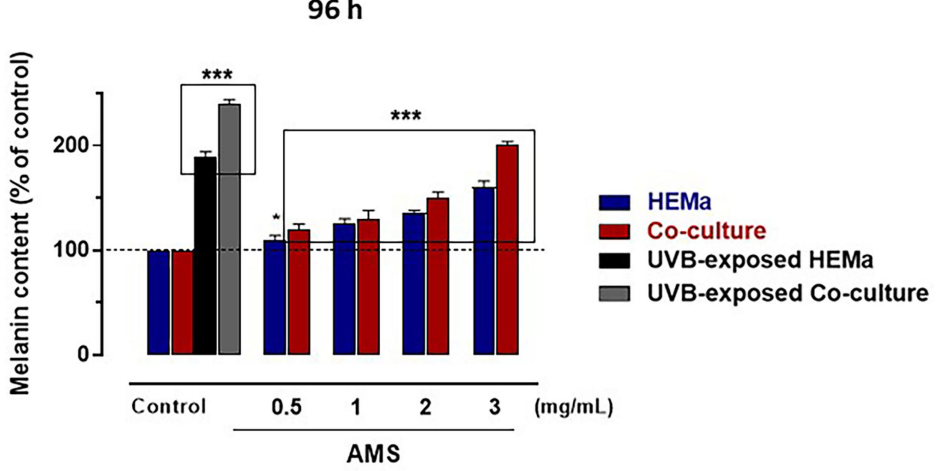

Figure 2 Total melanin content assay in HEMa cells and HEMa-HHFK co-cultures by spectrophotometric assay following 48 (A) and $96 \mathrm{~h}$ (B) of incubation in vitro with the indicated concentrations of AMS $(0.5 \div 3 \mathrm{mg} / \mathrm{mL})$, or after UVB irradiation under controlled conditions $\left(40 \mathrm{~mJ} / \mathrm{cm}^{2}\right)$, as described in the experimental section. Results are normalized based on cell count. ${ }^{* * *} \mathrm{p}<0.00 \mathrm{I}$ vs control (untreated cells); ${ }^{*} \mathrm{p}<0.0 \mathrm{I}$ vs control (untreated cells).

were considerably more intense when melanocytes were in coculture with keratinocytes. Indeed, in the latter case total quantities of melanin almost 2-fold higher than those of control cells were detected. Overall, both in pure primary melanocyte cultures and in co-culture systems, the nutraceutical supplement triggers an important promelanogenic effect.

\section{AMS Upregulates Tyrosinase Expression in Human Melanocytes}

Considering the promelanogenic effects of the nutraceutical preparation observed in human melanocytes, we subsequently analysed the expression of tyrosinase, the main regulatory enzyme along the biosynthetic pathway of melanins. Under the same experimental conditions described above, immunodetection presented in Figure $3 \mathrm{~A}$ and $\mathrm{B}$ underscores a significant increase in cytosolic levels of tyrosinase after incubation with AMS. In line with previous results, this increase is found in both pure and mixed cultures and it is dose- and time-dependent. Therefore, tyrosinase up-regulation appears to be a consequence of the incubation in vitro with the nutraceutical preparation. Exposure to UV-B light of the same melanocytes was used as a positive control, considered the main factor underlying the increase in the expression and activity of the tyrosinase enzyme.

A

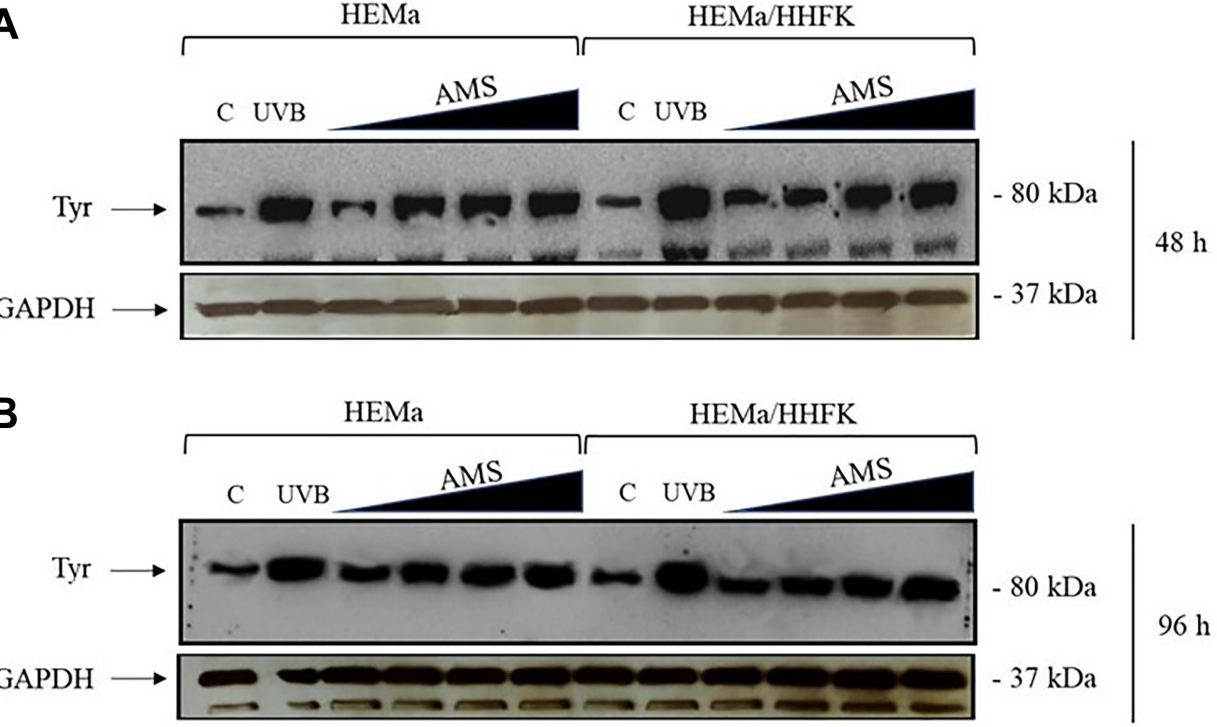

Figure 3 Tyrosinase (Tyr) expression study by immunodetection analysis in HEMa and HEMa-HHFK co-cultures incubated for 48 (A) and $96 \mathrm{~h}$ (B) with different concentrations of AMS supplement $(0.5, \mathrm{I}, 2$, and $3 \mathrm{mg} / \mathrm{mL})$ or irradiated by UVB under controlled conditions. Membranes were incubated with I: $250 \mathrm{monoclonal}$ tyrosinase antibody. Images are representative of four independent experiments and are cropped from original blots, as explicit by using clear delineation with dividing lines and white space (original acquisitions for Western blot experiments are shown in Figure SI). The anti-GAPDH antibody was used to standardize the amounts of proteins in each lane. 


\section{AMS Directly Promotes DHI Autoxidation and Melanin Formation}

Finally, based on pigment accumulation in melanocytes following incubation in vitro with AMS, we investigated in test tube the possible impact of the nutraceutical formulation on the chemical paths leading to melanin pigments by means of ad hoc spectrophotometric assays. The oxidative path from tyrosine to melanin involves several steps and two typical chromophoric phases. The early one, named dopachrome, is associated to the oxidation of the cyclic DOPA derivative and is described by the characteristic absorption band at $475 \mathrm{~nm}$. The later chromophoric phase, named melanochrome, features a much broader absorption at $560 \mathrm{~nm}$ which rapidly evolves into the typical featureless monotone UV-vis profile of melanin pigments and is associated to the oxidative polymerization of DHI. The melanochrome formation was followed in the progress of DHI autoxidation, up to 24 $\mathrm{h}$ reaction course, in presence of AMS, quercetin (here used as antioxidant) and without additives as blank. The choice of investigating the DHI to melanochrome conversion allowed to focus on the impact of the nutraceutical formulation at the level of the spontaneous chemical steps of melanin formation, reducing the participation of extracts-enzyme interactions which could have occurred monitoring the in vivo whole tyrosinase catalysed melanogenesis. Figure 4 collects the UV-vis profile evolution after DHI autoxidation alone and in presence of AMS and quercetin. Absorption profiles of autoxidation carried out in presence of $0.6 \mathrm{mg} / 100 \mathrm{~mL}$ of AMS (inset a) clearly witness an increased contribution of the chromophoric phase at late reaction times if compared to the blank. Moreover, as expected, the presence of quercetin $(0.6 \mathrm{mg} / 100 \mathrm{~mL})$, known to possess antioxidant properties, has the effect to slow down the oxidation progress, as witnessed by the lower absorption intensity observed in the visible region (inset c) with respect to the blank at the same reaction times.

\section{Discussion}

In the last decade boosting promotion of bioactive nutraceutical has underscored the importance of wellness, but also of a "nice look" simply for fashion or to postpone as much as possible the aging process. In this perspective, it is placed the continuous search for new natural products capable of acting at the skin level by improving and/or preserving our visual appearance. However, still nowadays numerous skin diseases based on pigmentation alterations can have serious social impacts, some of these being

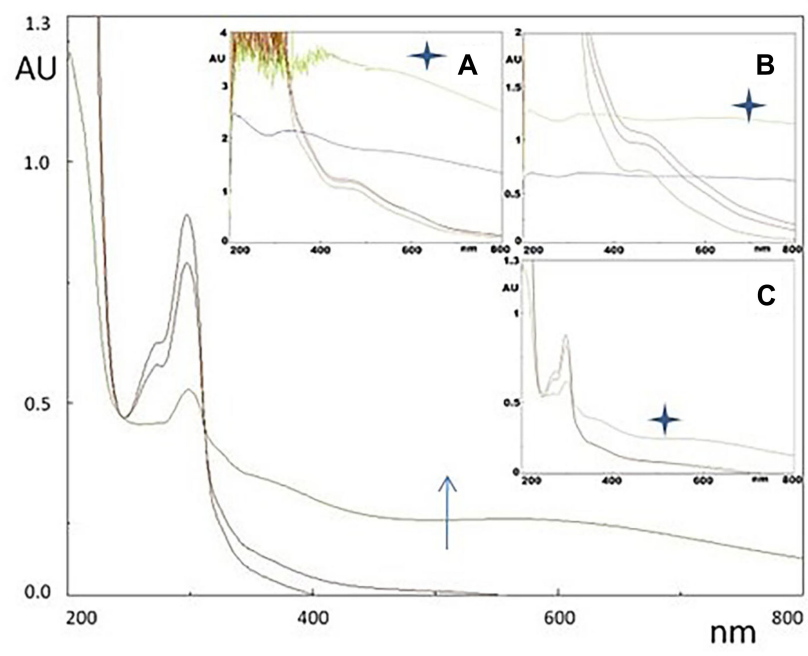

Figure 4 UV-vis profiles of $\mathrm{DHI}(6 \mathrm{mg} / 100 \mathrm{~mL} \mathrm{pH} 7.0)$ autoxidation (arrow denotes time progress, stars denote $24 \mathrm{~h}$ reaction time) along the process of melanin pigment formation. The broad maximum at around $600 \mathrm{~nm}$ denotes melanochrome formation. In the insets, the same process is monitored in presence of A) AMS formulation $(0.6 \mathrm{mg} / 100 \mathrm{~mL})$, C) quercetin $(0.6 \mathrm{mg} / 100 \mathrm{~mL})$ as an internal antioxidant control, and $\mathbf{B}$ ) plot is the blank.

disconcerting and still without remedy such as vitiligo. ${ }^{37,38}$ Consequently, disposal of skin pigmenting agents with both drug- and cosmetic-like properties is an urgent need, so that the discovery and development of melanogenesis stimulators have become a very dynamic area of research for academic and medical institutions, and cosmetic industries. ${ }^{39,40}$ Several recognized melanin activators are of synthetic derivation, but many others originate from natural molecules, with plant kingdom as the main resource of compounds endowed with favourable bioactivities. ${ }^{19,20}$ In this framework, the number of molecules effectively acting as melanogenesis stimulators is increasing but still limited. ${ }^{23,30}$ Contrariwise, several downregulators of melanogenesis endowed with a skin whitening/depigmenting action have been so far developed to treat hyperpigmentation disorders (eg, melasma and age spots). ${ }^{39}$ They act by interference with the melanogenesis process, mostly via tyrosinase inhibition, among others. $^{40,41}$ Moving in this direction, here we show that a natural formulation (AMS) from Annurca Apple cultivar, autochthonous of Campania region (Southern Italy) and endowed with a special tropism for human skin, stimulates a significant promelanogenic effect in pigmented cells. Indeed, without the evidence of adverse biological responses in vitro, a significant increase in melanin cytosolic accumulation is detected when human melanocytes are incubated under controlled conditions with the nutraceutical. Consistent with our findings, this effect is mainly 
due to an increased production of melanins in melanosomes via the complex melanogenetic pathway, with a resulting significant pigment accumulation at the level of the melanocytes. Additionally, a direct action on the chemical pathway leading to mature melanin granules via autoxidation of molecular precursors present in pigmented cells, such as DHI, seems to be effective. Assumed the antioxidant properties exhibited by the AMS formulation and based on experiments performed with quercetin which counteracts the autoxidation process in test tube, this last observation suggests that AMS effects onto the DHI autoxidation have to be ascribed to factors other than those of redox nature..$^{25,42}$ It may be speculated that, despite the likely presence of antioxidant components in the AMS formulation, prooxidant contribution at the level of DHI oxidative polymerization does operate producing an increase of detectable chromophoric phase due to melanin formation. According to our findings, some investigations have shown promelanogenic effect by molecules with polyphenolic structures. ${ }^{19,20}$ Indeed, some flavonoids from natural sources (eg, extracts of Salvia officinalis L.) can significantly increase melanin content, and further comprehensive studies revealed these natural compounds as capable of inducing expression and activity of the major melanogenetic proteins. $^{20,23}$ As a downside, other polyphenols including flavonoids or stilbenoids, have shown to affect tyrosinase expression/activity. ${ }^{39-41}$ Overall, the relevance of the promelanogenic effect prompted by AMS nutraceutical formulation can be underlined by the comparison with UV-induced melanin synthesis and accumulation, the foremost factor responsible for melanogenesis as part of the photoprotective tanning response of human skin. ${ }^{43}$ It is well known, in fact, that irradiation with UV light noticeably stimulates both expression and activity of tyrosinase, involved in the rate-limiting step during melanin production, ie, conversion of tyrosine in DOPA. Induction of melanin in UVB-exposed human melanocytes is largely mediated via the activation of the p38/MSK1/ CREB/MITF pathway. ${ }^{44,45}$ However, it can be triggered by a multiplicity of paracrine cytokines (eg, melanocytestimulating hormone (MSH), endothelin-1 (ET-1), and nitric oxide) through diverse signalling pathways converging on the activation of the microphthalmia-associated transcription factor (MITF), which regulates tyrosinase expression. ${ }^{14-18}$ Very recently, we have demonstrated that an oral consumption of $800 \mathrm{mg}$ in two administration a day of AMS for two months induces considerable effects on hair follicles, promoting skin and hair trophism. ${ }^{25,26,46}$
According to literature reports, these effects are probably due to oligomeric procyanidins, contained in significant quantities within the nutraceutical formulation and concerning to the broader class of bioflavonoids. ${ }^{47}$ In line with the tropism for skin, we now have shown this nutraceutical as endowed with an additional significant stimulatory bioactivity on melanocytes demonstrated by selected bioscreens throughout preclinical evaluations, thereby enlightening the hair re-pigmentation experienced by patients during clinical trials for the evaluation of some of its biological properties. ${ }^{26-28}$ Concentrations used to perform study in vitro $(0.5-3 \mathrm{mg} / \mathrm{mL})$ arise from former experiments designed to clarify the effects of the nutraceutical formulation on human cells. ${ }^{25,26}$ By high in vitro concentrations (up to $3 \mathrm{mg} / \mathrm{mL}$ ), we have assessed the formulation for adverse cellular effects, thereby validating its full biocompatibility. Conversely, studies on melanins production have been carried out at lower concentrations in vitro to possibly envision the real concentrations at the site of action in vivo after an oral administration of 800-1000 mg/day. Following this path, new pharmacokinetic experiments are underway by both models in vivo and clinical trials to monitor blood levels of the active ingredients, as well as concentrations at the sites of action. In this framework, very interesting is the data emerging from melanocytes-keratinocytes co-cultures model of human skin, where the presence of keratinocytes, like the physiological condition in vivo, further stimulates pigmented cells to produce melanins after treatment with AMS. Indeed, it is now believed that keratinocytes, the most abundant cell types of epidermis, are very sensitive to external stimuli. Under definite conditions (including UV light exposure), they can release several intrinsic factors, including melanogenic cytokines engaged in autocrine and paracrine signalling. ${ }^{17,18,43}$ Evidently, concurrent AMSdependent stimulation of keratinocytes could promote signalling cascade mechanisms playing a role in the upregulation of the melanogenic process, including expression of proteins involved in pigment production. Among these tyrosinase, a copper-containing $75 \mathrm{kDa}$ glycoprotein, extensively controls the production of melanin. ${ }^{48}$ After its synthesis in the endoplasmic reticulum and processing in the Golgi, tyrosinase is transferred to melanosomes. ${ }^{49}$ As in the case of UV light irradiation, our experimental data suggest that AMS biological effect on melanogenesis is accomplished by an up-regulation of tyrosinase expression. Nowadays evidence convincingly suggests that the stimulation of melanogenesis and of tyrosinase gene is 
largely mediated via the activation of MITF, in the context of an extremely complex dynamic process controlled by several factors. ${ }^{50}$ In addition, the regulation of tyrosinase expression by AMS is influenced by the presence of keratinocytes, reinforcing the concept of cellular crosstalk that underlies the existence of an intricate molecular control mechanism for melanogenesis. ${ }^{11,14,17,51}$ To better clarify the molecular basis of AMS bioactivity on melanogenesis, targeted experiments are in progress by advanced preclinical models. Henceforth, to fully exploit the beneficial properties of this natural formulation, indepth evidence will be required to shed light on precise mechanisms of actions and molecular targets. This would allow for further developments in the perspective of a novel drug-like agent for the care of skin disorders caused by hypopigmentation, as well as to counteract alterations by photoaging.

\section{Acknowledgment}

This study was supported by NGN Healthcare New Generation Nutraceuticals srl.

\section{Author Contributions}

Overall, all authors made a significant contribution to the work reported, whether that is in the conception, study design, execution, acquisition of data, analysis and interpretation, or in all these areas; took part in drafting, revising or critically reviewing the article; gave final approval of the version to be published; have agreed on the journal to which the article has been submitted; and agree to be accountable for all aspects of the work. Maria Grazia Ferraro and Marialuisa Piccolo are co-first authors. Carlo Irace and Ettore Novellino are co-last authors.

\section{Disclosure}

The authors declare no conflicts of interest for this work.

\section{References}

1. Slominski A, Tobin DJ, Shibahara S, Wortsman J. Melanin pigmentation in mammalian skin and its hormonal regulation. Physiol Rev. 2004;84(4):1155-1228.

2. Solano F. Melanins: skin pigments and much more - types, structural models, biological functions, and formation routes. New Journal of Science. 2014;2014:1-28. doi:10.1155/2014/498276

3. Maranduca MA, Branisteanu D, Serban DN, et al. Synthesis and physiological implications of melanic pigments. Oncol Lett. 2019;17 (5):4183-4187. doi:10.3892/ol.2019.10071

4. Schlessinger DI, Anoruo M, Schlessinger J. Biochemistry, Melanin. Treasure Island (FL): StatPearls Publishing; 2020.

5. Watt AAR, Bothmab JP, Meredith P. The supramolecular structure of melanin. Soft Matter. 2009;5:3754-3760. doi:10.1039/b902507c
6. Panzella L, Ebato A, Napolitano A, Koike K. The late stages of melanogenesis: exploring the chemical facets and the application opportunities. Int $J$ Mol Sci. 2018;19(6):1753. doi:10.3390/ ijms 19061753

7. Ortonne JP. Photoprotective properties of skin melanin. Br J Dermatol. 2002;146(61):7-10. doi:10.1046/j.1365-2133.146.s61.3.x

8. Simon JD, Peles DN. The red and the black. Acc Chem Res. 2010;43 (11):1452-1460. doi:10.1021/ar100079y

9. Cichorek M, Wachulska M, Stasiewicz A, Tymińska A. Skin melanocytes: biology and development. Postepy Dermatol Alergol. 2013;30(1):30-41. doi:10.5114/pdia.2013.33376

10. D'Alba L, Shawkey MD. Melanosomes: biogenesis, properties, and evolution of an ancient organelle. Physiol Rev. 2019;99(1):1-19. doi:10.1152/physrev.00059.2017

11. Seiberg M. Keratinocyte-melanocyte interactions during melanosome transfer. Pigment Cell Res. 2001;14(4):236-242. doi:10.1034/j.16000749.2001.140402.x

12. Schallreuter KU, Kothari S, Chavan B, Spencer JD. Regulation of melanogenesis-controversies and new concepts. Exp Dermatol. 2008;17(5):395-404. doi:10.1111/j.1600-0625.2007.00675.x

13. Pavan WJ, Sturm RA. The genetics of human skin and hair pigmentation. Annu Rev Genomics Hum Genet. 2019;20(1):41-72. doi:10.1146/annurev-genom-083118-015230

14. D’Mello SA, Finlay GJ, Baguley BC, Askarian-Amiri ME. Signaling pathways in melanogenesis. Int $J$ Mol Sci. 2016;17(7):1144. doi:10.3390/ijms 17071144

15. Serre C, Busuttil V, Botto JM. Intrinsic and extrinsic regulation of human skin melanogenesis and pigmentation. Int $J$ Cosmet Sci. 2018;40(4):328-347. doi:10.1111/ics.12466

16. Slominski A. Neuroendocrine activity of the melanocyte. Exp Dermatol. 2009;18(9):760-763. doi:10.1111/j.1600-0625.2009.00892.x

17. Yuan XH, Jin ZH. Paracrine regulation of melanogenesis. $\mathrm{Br}$ J Dermatol. 2018;178(3):632-639. doi:10.1111/bjd.15651

18. Rzepka Z, Buszman E, Beberok A, Wrześniok D. From tyrosine to melanin: signaling pathways and factors regulating melanogenesis. Postepy Hig Med Dosw. 2016;70:695-708. doi:10.5604/ 17322693.1208033

19. Zaidi KU, Ali SA, Ali AS, Thawani V. Natural melanogenesis stimulator a potential tool for the treatment of hypopigmentation disease. Int J Mol Biol. 2017;2(1):37-40.

20. Niu C, Aisa HA. Upregulation of melanogenesis and tyrosinase activity: potential agents for vitiligo. Molecules. 2017;22(8):1303. doi:10.3390/molecules 22081303

21. Niu C, Yin L, Nie LF, et al. Synthesis and bioactivity of novel isoxazole chalcone derivatives on tyrosinase and melanin synthesis in murine B16 cells for the treatment of vitiligo. Bioorg Med Chem. 2016;24(21):5440-5448. doi:10.1016/j.bmc.2016.08.066

22. Chisvert A, Benedé JL, Salvador A. Chapter 6 - tanning and whitening agents in cosmetics: regulatory aspects and analytical methods. In: Salvador A, Chisvert A, editors. Analysis of Cosmetic Products. 2nd ed. Elsevier; 2018:107-121.

23. Ali SA, Naaz I, Zaidi KU, Ali AS. Recent updates in melanocyte function: the use of promising bioactive compounds for the treatment of hypopigmentary disorders. Mini Rev Med Chem. 2017;17 (9):785-798. doi:10.2174/1389557516666161223153953

24. Boo YC. Emerging strategies to protect the skin from ultraviolet rays using plant-derived materials. Antioxidants. 2020;9(7):637. doi:10.3390/antiox 9070637

25. Piccolo M, Ferraro MG, Maione F, et al. Induction of hair keratins expression by an annurca apple-based nutraceutical formulation in human follicular cells. Nutrients. 2019;11(12):3041. doi:10.3390/ nu11123041

26. Tenore GC, Caruso D, Buonomo G, et al. Annurca apple nutraceutical formulation enhances keratin expression in a human model of skin and promotes hair growth and tropism in a randomized clinical trial. J Med Food. 2018;21(1):90-103. doi:10.1089/jmf.2017.0016 
27. Tenore GC, Caruso D, Buonomo G, et al. A healthy balance of plasma cholesterol by a novel annurca apple-based nutraceutical formulation: results of a randomized trial. J Med Food. 2017;20 (3):288-300. doi:10.1089/jmf.2016.0152

28. Tenore GC, Caruso D, Buonomo G, et al. Lactofermented annurca apple puree as a functional food indicated for the control of plasma lipid and oxidative amine levels: results from a randomised clinical trial. Nutrients. 2019;11(1):122. doi:10.3390/nu11010122

29. Yamauchi K, Mitsunaga T. Methylquercetins stimulate melanin biosynthesis in a three-dimensional skin model. J Nat Med. 2018;72 (2):563-569. doi:10.1007/s11418-018-1175-0

30. Takekoshi S, Nagata H, Kitatani K. Flavonoids enhance melanogenesis in human melanoma cells. Tokai J Exp Clin Med. 2014;39 (3):116-121.

31. Piccolo M, Misso G, Ferraro MG, et al. Exploring cellular uptake, accumulation and mechanism of action of a cationic Ru-based nanosystem in human preclinical models of breast cancer. Sci Rep. 2019;9 (1):7006. doi:10.1038/s41598-019-43411-3

32. Iovine B, Nino M, Irace C, Bevilacqua MA, Monfrecola G. Ultraviolet $\mathrm{B}$ and $\mathrm{A}$ irradiation induces fibromodulin expression in human fibroblasts in vitro. Biochimie. 2009;91(3):364-372. doi:10.1016/j.biochi.2008.10.017

33. Song X, Mosby N, Yang J, Xu A, Abdel-Malek Z, Kadekaro AL. alpha-MSH activates immediate defense responses to UV-induced oxidative stress in human melanocytes. Pigment Cell Melanoma Res. 2009;22(6):809-818. doi:10.1111/j.1755-148X.2009.00615.x

34. Hosoi J, Abe E, Suda T, Kuroki T. Regulation of melanin synthesis of B16 mouse melanoma cells by 1 alpha, 25-dihydroxyvitamin D3 and retinoic acid. Cancer Res. 1985;45(4):1474-1478.

35. Irace C, Misso G, Capuozzo A, et al. Antiproliferative effects of ruthenium-based nucleolipidic nanoaggregates in human models of breast cancer in vitro: insights into their mode of action. Sci Rep. 2017;7(1):45236. doi:10.1038/srep45236

36. Santamaria R, Fiorito F, Irace C, et al. 2,3,7,8-Tetrachlorodibenzop-dioxin impairs iron homeostasis by modulating iron-related proteins expression and increasing the labile iron pool in mammalian cells. Biochim Biophys Acta. 2011;1813(5):704-712. doi:10.1016/j. bbamcr.2011.02.003

37. Yamaguchi Y, Hearing VJ. Melanocytes and their diseases. Cold Spring Harb Perspect Med. 2014;4(5):a017046. doi:10.1101/cshperspect.a017046

38. Harris J. Vitiligo's impact is in the eye of the beholder. Nature. 2018;563(7732):S99. doi:10.1038/d41586-018-07435-5

39. Pillaiyar T, Manickam M, Jung SH. Downregulation of melanogenesis: drug discovery and therapeutic options. Drug Discov Today. 2017;22(2):282-298. doi:10.1016/j.drudis.2016.09.016
40. Boo YC. Up- or downregulation of melanin synthesis using amino acids, peptides, and their analogs. Biomedicines. 2020;8(9):E322. doi:10.3390/biomedicines8090322

41. Lajis AFB, Ariff AB. Discovery of new depigmenting compounds and their efficacy to treat hyperpigmentation: evidence from in vitro study. J Cosmet Dermatol. 2019;18(3):703-727. doi:10.1111/ jocd. 12900

42. Chan CF, Wu CT, Huang WY, et al. Antioxidation and melanogenesis inhibition of various dendrobium tosaense extracts. Molecules. 2018;23(7):1810. doi:10.3390/molecules23071810

43. Nguyen NT, Fisher DE. MITF and UV responses in skin: from pigmentation to addiction. Pigment Cell Melanoma Res. 2019;32 (2):224-236. doi:10.1111/pcmr.12726

44. Tagashira H, Miyamoto A, Kitamura S, et al. UVB stimulates the expression of endothelin B receptor in human melanocytes via a sequential activation of the p38/MSK1/CREB/MITF pathway which can be interrupted by a French Maritime Pine Bark Extract through a direct inactivation of MSK1. PLoS One. 2015;10(6): e0128678. doi:10.1371/journal.pone.0128678

45. Terazawa S, Imokawa G. Signaling cascades activated by UVB in human melanocytes lead to the increased expression of melanocyte receptors, endothelin B Receptor and c-KIT. Photochem Photobiol. 2018;94(3):421-431. doi:10.1111/php.12848

46. Badolati N, Sommella E, Riccio G, et al. Annurca apple polyphenols ignite keratin production in hair follicles by inhibiting the pentose phosphate pathway and amino acid oxidation. Nutrients. 2018;10 (10):1406. doi:10.3390/nu10101406

47. Takahashi T, Kamiya T, Hasegawa A, Yokoo Y. Procyanidin oligomers selectively and intensively promote proliferation of mouse hair epithelial cells in vitro and activate hair follicle growth in vivo. $J$ Invest Dermatol. 1999;112(3):310-316. doi:10.1046/j.15231747.1999.00532.x

48. Kanteev M, Goldfeder M, Fishman A. Structure-function correlations in tyrosinases. Protein Sci. 2015;24(9):1360-1369. doi:10.1002/ pro. 2734

49. Jimbow K, Park JS, Kato F, et al. Assembly, target-signaling and intracellular transport of tyrosinase gene family proteins in the initial stage of melanosome biogenesis. Pigment Cell Res. 2000;13 (4):222-229. doi:10.1034/j.1600-0749.2000.130403.x

50. Gilchrest BA, Eller MS. DNA photodamage stimulates melanogenesis and other photoprotective responses. J Investig Dermatol Symp Proc. 1999;4(1):35-40. doi:10.1038/sj.jidsp.5640178

51. Nordlund JJ. The melanocyte and the epidermal melanin unit: an expanded concept. Dermatol Clin. 2007;25(3):271-281. doi:10.1016/ j.det.2007.04.001
Clinical, Cosmetic and Investigational Dermatology is an international, peer-reviewed, open access, online journal that focuses on the latest clinical and experimental research in all aspects of skin disease and cosmetic interventions. This journal is indexed on CAS.
The manuscript management system is completely online and includes a very quick and fair peer-review system, which is all easy to use. Visit http://www.dovepress.com/testimonials.php to read real quotes from published authors. 